\title{
Characterization of CMOS-MEMS Resonant Pressure Sensors
}

\author{
Saoni Banerji, Daniel Fernández, and Jordi Madrenas
}

\section{1}

Abstract - Comprehensive characterization results of a CMOSMEMS resonant pressure sensor are presented. We have extensively evaluated the key performance parameters of our device in terms of quality factor $(Q)$ variations under variable conditions of temperature and pressure, characterized by Knudsen number $\left(K_{n}\right)$. The fundamental frequency of the reported device is $104.3 \mathrm{kHz}$. Over the full-scale pressure range of 0.1 to 100 $\mathrm{kPa}$ and a temperature range of $-10{ }^{\circ} \mathrm{C}$ to $85^{\circ} \mathrm{C}, Q$ from 450 to 62.6 have been obtained. Besides, static variations of the device capacitance have been measured and analyzed with temperature to evaluate the spring softening and the pull-in effects. A nonlinearity analysis has been performed to assess the device stability. Furthermore, a statistical mismatch analysis has been carried out to determine the deviation of resonance with etching time and ascertain maximum device yield. With our in-house BEOL metal-layer release, this sensor can be monolithically embedded in the same substrate as standard CMOS integrated circuits, resulting in a significant cost and area reduction.

Index Terms-CMOS-MEMS resonator, Quality factor, Knudsen number, Pressure sensor, MEMS characterization, Statistical mismatch, Nonlinearity

\section{INTRODUCTION}

$I^{\mathrm{N}}$ advanced societies, microelectromechanical systems (MEMS) represent a significant segment of the market dominated by consumer electronics [1]. This market has not been widely adopting pressure sensors in wearables due to an involvement of cost and volume of additional sensor components. Integration, i.e. embedding sensors and electronics in a single chip, is the primary strategy to achieve a widespread adoption of pressure sensors. One of the current challenges of integration technology posed by the international roadmap for the semiconductor industry is the modification and standardization of CMOS technology to accommodate MEMS technology i.e. to integrate sensors and electronics in a single chip combining different fabrication techniques [2]. The advent of monolithically integrated CMOS-MEMS resonant pressure sensors have resolved this issue and can be targeted for the consumer market for assisted GPS (Global Positioning Systems) in indoor navigation systems, altimeters, and other possible interesting applications [2]. The significance of

\footnotetext{
1 An earlier reduced version of this paper was presented at the IEEE Sensors 2016 Conference in Orlando, Florida and was published in its proceedings:

http://ieeexplore.iee.. rg/stamp/stamp.jsp?tp=\&arnumber=7808403\&isnumbe $\mathrm{r}=7808393$

This work has been partially funded by the Spanish Ministry of Science and Innovation and the European Social Fund (ESF) under Project TEC201567278-R. Saoni Banerji holds an FI scholarship funding by the Catalan government and European Social Fund (ESF).
}

MEMS integrated with CMOS stems from their many advantages, i.e. reduced cost, small size, continuous miniaturization, high performance, and reliability while reasonably maintaining their key performance parameters [1].

Several CMOS-MEMS devices have been implemented by using post-CMOS processing such as inertial sensors, magnetometers, pressure sensors, etc. [3]-[6]. The capacitive MEMS pressure sensors are the most widely used pressure sensor types, as they show excellent noise performance, exhibit low power consumption and permit easy integration with processing circuitry [4], [6], [7].

Technological efforts have already been made towards the integration of both CMOS and MEMS. For example, Narducci et al. reported a capacitive pressure sensor using Al metal layers of a commercial CMOS process as sensing electrodes [6]. Cheng et. al. used multiple metal layers to form the mechanical structure of a CMOS based pressure sensor to improve the sensitivity [4].

In order to explore the commercial feasibility of CMOSMEMS pressure sensors, comprehensive characterization results are required. So far, several characterization results of MEMS pressure sensors have been reported. A $9.61 \mathrm{~mm}^{2} \mathrm{SOI}$ micromachined resonant pressure sensor with capacitive readout packaged in the dry air has been developed and characterized by Sen et al. [8]. This double-ended tuning fork resonator was characterized over a full-scale pressure range of 100 to $400 \mathrm{kPa}$ and a temperature range of $-10{ }^{\circ} \mathrm{C}$ to $85^{\circ} \mathrm{C}$, which has a fundamental frequency of $34.55 \mathrm{kHz}$ and Quality factor $(Q)$ ranging from 1146 to 1772 . Montiel-Nelson et. al [9] developed a $0.36 \mathrm{~mm}^{2}$ capacitive detected diaphragm sensor using Metal Mumps process characterized under the range of 0-30 $\mathrm{kPa}$. The device exhibited a resonance frequency of 4 $\mathrm{MHz}$ and achieved a low power consumption of $16.56 \mu \mathrm{W}$ in combination with a capacitance-to-digital converter fabricated on CMOS standard process. Sundararajan et. al [10] developed a CMOS integrated capacitive pressure sensor, fabricated in 0.18 um SiGe MEMS process, with the sensor held and linked to the CMOS beneath. The $0.56 \mathrm{~mm}^{2}$ elliptic structured diaphragm was characterized over a pressure range of 10 to 100

Saoni Banerji and Daniel Fernández are with Nanusens, CENT-Parc Tecnològic del Vallès, Av. del Parc Tecnològic, 3, 08290 Cerdanyola Del Vallès, Spain

Jordi Madrenas is with Electronic Engineering Department, Universitat Politècnica de Catalunya, Jordi Girona 1-3, 08034 Barcelona, Spain. 


\section{$>$ REPLACE THIS LINE WITH YOUR PAPER IDENTIFICATION NUMBER (DOUBLE-CLICK HERE TO EDIT) $<$}

$\mathrm{kPa}$ and has shown a wider pressure sensing range at a lower supply voltage compared to commercial pressure sensors. Kuo et. al [11] developed a $0.119 \mathrm{~mm}^{2}$ two comb-finger structured temperature and pressure sensor based on $Q$-factor using a 0.18 $\mu \mathrm{m}$ 1P6M CMOS ASIC/MEMS process adopted to monolithically integrate MEMS sensor and circuits, and characterized over a pressure range of $0.1-1.6 \mathrm{kPa}$. The device shows a resonant frequency of $41.1 \mathrm{kHz}$ and $\mathrm{Q}$ ranging from 2600 to 450 under the characterized pressure range and it was fabricated using an anisotropic silicon oxide etching (DRIE), which incurs a higher potential cost than that for the reported device presented in this work.

The present work is dedicated to the extensive characterization of a $0.0196 \mathrm{~mm}^{2}$ CMOS-MEMS resonant pressure sensor manufactured in a standard $250 \mathrm{~nm}$ CMOS technology. We have implemented our device as a two-metal layer lattice suspended on short folded metal beams so that it resonates at air pressure with a reasonable $Q$. The presented approach has shown significant advantages in terms of $\mathrm{C}-\mathrm{SWaP}$ (Cost, Size, Weight, and Power) compared to the other approaches. Owing to the reduction in size compared to the resonator size specified in the current state of the art, and their complete integration with CMOS circuitry, our device focusses in low cost and high-volume production, enabling their wide use in Human Interface Devices (HIDs). From the convergence of the measurement data to the behavior of the second order resonator model with temperature, pressure, electrical conditions, non-linearity and statistical mismatch, the reported characterization methodology allows a designer, to envision several aspects, for optimal functioning of CMOS-MEMS resonators. Some details of the device design and fabrication are discussed in Section II. Section III entails the methodology adapted to perform the device characterization, including the effect of temperature and pressure in addition to the studies concerning nonlinearity and statistical mismatch. An interpretation of their results has also been presented. This is followed by concluding remarks in Section IV.

\section{DEVICE DESIGN AND FABRICATION}

The cross-section diagram of a parallel plate capacitor illustrated in Fig. 1, is employed for our CMOS-MEMS resonator design. When a voltage is applied to the movable membrane, an electrostatic force works to reduce the plate separation between the movable membrane and fixed bottom metal plate from $z_{0}$ to $z_{0}-z$. The electrostatic voltage is countered by the spring force, $F=-k z$ at small voltages. However, as the voltage is increased, the plates eventually snap together, popularly known as the pull-in effect. The second-order differential equation for the lumped resonator model can be expressed in Equation (1) as

$m \ddot{z}+b \dot{z}+k z=\sum F_{\text {ext }}$ where $F_{\text {ext }}$ is the external force applied to the resonator, $m$ is the effective mass, $b$ is the net damping coefficient, and $k$ is the spring constant. Here $z, \dot{z}, \ddot{z}$ are the displacement, velocity, and acceleration of the system respectively [12].

An flexural mode resonator has been analytically developed and numerically validated by FEM models in COMSOL Multiphysics in order to optimize the Q sensitivity without affecting the device capacitance [12], [13].The pressure sensor design has an effective mass of $0.4 \mu \mathrm{g}$ with an optimal geometry of $140 \mu \mathrm{m} \times 140 \mu \mathrm{m} \times 8 \mu \mathrm{m}$ having $6 \times 6$ perforations along the row and column of the plate, respectively, to achieve maximum $Q$ while presenting an acceptable capacitance variation to the electronics[14].

The optimization procedure has been followed by manufacturing the resonator design in a $250 \mathrm{~nm}$ CMOS technology, which in general, can be migrated to other technologies. The device release has been performed at the dice level in the UPC-DEE cleanroom with a hydrogen fluoride based etchant to release the back-end of line (BEOL) metal layers. The release was followed by resist removal and a rinse in methanol to reduce stiction issues and then the samples were dried in the oven [15], [16]. The reported capacitive pressure sensor, fabricated using IHP SG25 process, is implemented as a two aluminum layers (with a thickness of $2 \mu \mathrm{m}$ and $3 \mu \mathrm{m}$ ) separated by a $3 \mu \mathrm{m}$ thick tungsten via. Fig. 2 shows the scanning electron microscope (SEM) micrograph of the fabricated test chip.

The reported device shows a quality factor of 70 and reduced damping coefficient of $4.34 \mu \mathrm{Ns} / \mathrm{m}$ at atmospheric pressure. More details on the device structure can be found in [12], [13].

\section{DEVICE CHARACTERIZATION AND RESUlTS}

The temperature and pressure conditions have been varied to investigate the performance of the pressure sensor in terms of $Q$. The device behavior has further been evaluated with the variations of the resonator-to-electrode gap in response to temperature and parametric excitations. A mismatch analysis was carried out to analyze the statistics of released samples at the same time. This has been followed by the characterization of nonlinearities and estimation of the maximum usable vibration amplitude to set the upper bound of the device signal strength.

\section{A. Characterization Set-up for the CMOS-MEMS pressure sensor}

The frequency response of the CMOS-MEMS pressure sensor was characterized using a custom-built experimental setup based on the Agilent 4294 A precision Impedance Analyzer, as illustrated in Fig. 3.

A pressure controller was built using a bell jar manually equipped with probes and an external microscope for proper placement of the test chip on a TEC1-12705 Peltier surface. A thermometer is attached to the Peltier module by means of thermal paste, that was connected to the probes attached to the device. Besides, the low mass of the device ascertains uniform 


\section{$>$ REPLACE THIS LINE WITH YOUR PAPER IDENTIFICATION NUMBER (DOUBLE-CLICK HERE TO EDIT) $<$}

chip temperature and excellent stability. This was externally connected to a powerful vacuum pump for generating and varying pressures from $100 \mathrm{~Pa}$ to $100 \mathrm{kPa}$ displayed on a digital vacuum gauge, that was capable of measuring pressures accurately below $50 \mathrm{kPa}$. The temperatures were varied using the hot and cold sides of the Peltier and a variable power supply from sub-zero temperatures of $-10{ }^{\circ} \mathrm{C}$ to high temperatures of $85{ }^{\circ} \mathrm{C}$.

The sensor performance was further evaluated from these analyses in terms of the quality factor and resonance frequency. They were correlated with temperature and pressure while the resonance frequency was used as a measure to evaluate the device stability under variable electrical stimulations i.e. DC bias and drive levels.

\section{B. Device characterization under environmental conditions}

A candidate test chip with a resonance frequency of 104.3 $\mathrm{kHz}$ has been chosen for all our measurements.

Knudsen number, $K_{n}$ is an important parameter that classifies the degree of rarefaction of gases into four flow regimes, depending on the device gap i.e. the surrounding temperature and pressure [13], [16], [17]. It can be expressed as

$K_{n}=\lambda / h_{0}=K_{B} T / \sqrt{2} \pi d^{2} P h_{0}$

where $K_{B}$ is the Boltzmann constant $(1.38 \mathrm{e}-23 \mathrm{~J} / \mathrm{K}), d$ is the mean molecular diameter of the molecule $\left(3.7 \times 10^{-10} \mathrm{~m}\right), \lambda$ is the mean free path of the molecule, $T$ and $P$ are the operating temperature and pressure respectively while $h_{0}$ is the characteristic device length, which is $\mathrm{z}_{0}=2.5 \mu \mathrm{m}$, in our case [14]. In Equation (2), $K_{n}$ has been used to characterize both pressure and temperature, which in turn has characterized the device performance in terms of $Q$, discussed in the following sub-sections. An in-detail information on the significance of the Knudsen number can be found in [13].

\section{1) Pressure Measurements}

The $Q$ of a resonating pressure sensor is primarily dependent on the viscosity of the surrounding fluid i.e. air, in regimes where air damping is the dominant loss mechanism.

Fig. 4 shows the frequency response of the device. It was recorded from $100 \mathrm{~Pa}$ to atmospheric pressure, at room temperature, i.e. $25^{\circ} \mathrm{C}$, and for a fixed bias voltage of $10 \mathrm{~V}$. The oscillatory excitation amplitude was limited to $30 \mathrm{mV}$ for all measurements to mitigate the nonlinearity effects due to excessive amplitude vibration. The device quality factor was obtained from the frequency response by aligning and validating them with the response of a second-order behavioral model using $Q$ as one of its inputs [13], [17]. The manufactured sensor was primarily operated in the slip flow and transition flow regimes, as obtained from the $K_{n}$ value. The device performance in these flow regimes depends on a combination of structural and film damping effects. In order to provide a brief overview of the correlation of device performance with pressure, the $K_{n}$ dependency on pressure for constant temperature becomes, from Equation (2):

$K_{n}\left(25^{\circ} \mathrm{C}\right)=0.0068 / P h_{0}=2720 / P$

where $h_{0}=z_{0}$ is $2.5 \times 10^{-6} \mathrm{~m}$. The $Q$-factors of the resonator over the full-scale pressure of $100 \mathrm{~Pa}-100 \mathrm{kPa}$ range from 450 to 70. It has been observed that as the pressure increases from 100 Pa to $1 \mathrm{kPa}, Q$ decreases by $23.33 \%$. When pressure increases from $1 \mathrm{kPa}$ to $10 \mathrm{kPa}$ in the transition flow regime where both structural damping and film damping effects are responsible for a loss of the device performance, $Q$ suffers a reduction of $68.12 \%$. Finally, when the pressure increases transits from the transition flow regime to slip flow regime, $Q$ reduces by $50 \%$. As the pressure ranges from molecular flow regime to transition flow regime, the device $Q$ suffers a higher reduction than within the pressures range in the transition flow regime. This occurs due to both material losses and film damping present in this pressure regime.

Following this, the extracted quality factors were plotted against varying pressures characterized by the $K_{n}$. As shown in Fig. 5, the manufactured sensor $Q$ follows an inverse power law with the surrounding pressures. The variation of $Q$ with pressure $\mathrm{P}$ is fitted as

$Q=a P^{b} \cong 2888 P^{-0.341}$

where a and b are coefficients relating $Q$ to $P$.

When $K_{n}$ is greater than 10 , the device behavior is in the molecular regime. The range of surrounding pressures is estimated to be under $272 \mathrm{~Pa}$, at room temperature, taking the electrode-to-resonator gap as $2.5 \mu \mathrm{m}$. The device suffers solely from structural damping effects in this pressure regime, as the mean free path of the molecule exceeds the resonator-toelectrode gap, neglecting the intermolecular collisions [17]. The variation of $Q$ follows a power law with the surrounding pressures in a portion of the transition flow and slip flow regime, where the constant of proportionality is -0.341 . However, this constant of proportionality varies in the molecular flow regime where the fluid damping ceases i.e. $Q$ is not demonstrated by fluid damping mechanisms of ambient pressure, but by mechanical losses. This is not considered in the analytical model used to fit the measurement data. Hence, for the molecular regime, the divergence is due to the structural damping alone. This explains the cause of divergence of the experimental values of $Q$ from fitted line. As the mean free path of the molecule becomes comparable to the electrode-toresonator gap, the $K_{n}$ ranges between 0.1 and 10. Surrounding pressure ranges between $272 \mathrm{~Pa}$ to $27.2 \mathrm{kPa}$. In this region, both structural damping and film damping effects play a significant role in the computation of $Q$. As the Knudsen number further reduces to fall in the range of 0.01 and 1 , with pressure ranges from $27200 \mathrm{~Pa}$ to pressures beyond atmospheric pressure, device losses occur mainly due to film-damping effects. 


\section{$>$ REPLACE THIS LINE WITH YOUR PAPER IDENTIFICATION NUMBER (DOUBLE-CLICK HERE TO EDIT) $<$}

\section{2) Thermal Measurements}

This section details the thermal variations to characterize the prototype resonator at a particular pressure in the transient region [18]. Temperature disturbance is a vital concern in the field of resonant pressure micro sensors. They can lead to frequency drift owing to the stress changes [18]-[22].

The device characterization has been performed from $-10{ }^{\circ} \mathrm{C}$ to $85^{\circ} \mathrm{C}$, in coherence with the consumer electronics standards. The pressure is kept constant at $10 \mathrm{kPa}$ i.e. in transition flow regime. This point is selected to observe distinct variations in resonance frequency in response to temperature variations. A $60 \mathrm{~W}$ Peltier module with variable voltage supply was used to set the temperature.

The observed effect of temperature on $Q$ of a microscale resonator in a low-pressure air damping regime can be modeled in first order approximation as:

$Q=E_{\text {stored }} / E_{\text {diss }}$

$=C_{S A} /(n \cdot \bar{v})=C_{S A} \frac{\sqrt{K_{B} T}}{P} \propto 1 / T^{\gamma}$

Here, $C_{S A}$ is the constant involving the surface area perpendicular to the direction of motion, the mode shape of the resonating membrane etc., $n$ is the density of molecules and $\bar{v}$ is the mean velocity of air molecules, $K_{B}$ is the Boltzmann constant, $\mathrm{T}$ and $\mathrm{P}$ are the temperature and pressure respectively, and $\gamma$ is the TCQ (Temperature coefficient of $Q$ ) that defines the inverse temperature dependence of the quality factor, depending on the pressure regime, characterized by $K_{n}$.

The Quality factor of micro-resonators due to air damping in kinetic gas regime depends on the resonator beam thickness, density, resonant frequency and mass of the resonator, as expressed in Equation (2) of [18]. Accordingly, the estimated value of $C_{S A}$ for our device is $2.58 \times 10^{5} \mathrm{kHzkg}^{0.5} / \mathrm{m}^{2}$.

Keeping the pressure constant, $K_{n}$ can be correlated with the surrounding temperature:

$$
\begin{aligned}
K_{n}(10 \mathrm{kPa})= & 2.46 \times 10^{-10} \mathrm{~T} / \mathrm{z}_{0} \\
& =9.8 \times 10^{-5} \mathrm{~T}
\end{aligned}
$$

Fig. 6 shows the device impedance module dependency on frequency under temperatures $-10,25$ and $85{ }^{\circ} \mathrm{C}$. The antiresonance is observed due to the parasitics of the device capacitance and the experimental setup. These measurements have been aligned to simulations with a second-order behavioral model to obtain the $Q^{\prime}$ s at varying temperatures. The experimental data fit well with the trend between resonator $Q$ and temperature in different pressure regimes, as suggested in Equation (5). The initial $Q$ had decreased to a factor of 0.74 when the temperature changed from $25^{\circ} \mathrm{C}$ to $85^{\circ} \mathrm{C}$, at $10 \mathrm{kPa}$ in the transition flow regime. As the temperatures were increased from sub zero temperatures of $-10{ }^{\circ} \mathrm{C}$ to $85^{\circ} \mathrm{C}$, the device $Q$ was observed to decrease from 85 to 62.6 i.e. the
TABLE I

DEVICE PARAMETERS

\begin{tabular}{llll}
\hline \hline Symbol & \multicolumn{1}{c}{ Quantity } & Values & Units \\
\hline$\beta$ & $\begin{array}{l}\text { Dimensionless } \\
\text { coefficient dependent } \\
\text { on shape of vibration } \\
\text { mode }\end{array}$ & 0.0784 & - \\
$T_{p}$ & $\begin{array}{l}\text { Device thickness } \\
L\end{array}$ & $8 \times 10^{-6}$ & $\mathrm{~m}$ \\
$E_{\text {avg }}$ & $\begin{array}{l}\text { Device length } \\
\text { Average Young's }\end{array}$ & $140 \times 10^{-6}$ & $\mathrm{~m}$ \\
$\rho_{\text {avg }}$ & $\begin{array}{l}\text { Average Density } \\
k\end{array}$ & 6500 & $\mathrm{GPa}$ \\
$z_{0}$ & $\begin{array}{l}\text { Spring coefficient } \\
\text { Resonator to electrode }\end{array}$ & $2.5 \times 10^{-6}$ & $\mathrm{~kg} / \mathrm{m}^{3}$ \\
$m$ & $\begin{array}{l}\text { Gap } \\
\text { Effective mass }\end{array}$ & $4.127 \times 10^{-10}$ & $\mathrm{~m} / \mathrm{m}$ \\
& & & $\mathrm{kg}$
\end{tabular}

experimental measurements report a reduction of $Q$ in a factor of 0.74 .

The fit of the extracted Q has been plotted as a function of $T$ and $K_{n}$ in Fig. 7, after extracting the device Q from the secondorder behavioral model with $\mathrm{T}$ as one of its inputs. For a pressure of $10 \mathrm{kPa}$, the values of the $K_{n}$ range from 0.24 to 0.33 where the air molecules behave as kinetic particles. Thus, the interaction between the device and the surrounding air is comparable to the intermolecular interactions. As a result, the structural and film damping effects play an equal role in the computation of the device $Q$. The observed trend suggests that with an increase in temperature, the device interactions rise along with the air-device interactions. This enhances the energy dissipation and reducing the net $Q$. The values $\gamma$ (TCQ)is 0.9 , that has been obtained from fitting the experimental frequency

response with the non-linear behavioral model, as shown in Fig. 7. The fitting line corresponds to Equation (5), that has been adapted to the behavioral model.Another important phenomenon is the dependence of the device resonance frequency on the temperature. For a flexural mode square diaphragm as in our case, the resonance frequency scales up as a function of the inverse of the main dimension squared and proportional to Young's modulus of the material used for the resonator, owing to the proportional relationship of $k$ and $E$ for aluminum. [21][22].

$f_{\text {nom }} \beta \frac{T_{p}}{L^{2}} \sqrt{\frac{E_{\text {avg }}}{\rho_{\text {avg }}}}=\frac{1}{2 \pi} \sqrt{\frac{k}{m}}$

where $f_{\text {nom }}$ is the normal frequency of a resonator at a nominal temperature $T_{0}$ with $V_{D C}=0 \mathrm{~V}$. $\beta$ is a dimensionless coefficient dependent on the shape of vibration mode; which in turn depends on the respective boundary conditions applicable to the structure, $L$ is the device length, $T_{p}$ is the device thickness, $E$ is the average young's modulus, and $\rho$ is the average material density. The geometrical parameters have been enlisted in 


\section{$>$ REPLACE THIS LINE WITH YOUR PAPER IDENTIFICATION NUMBER (DOUBLE-CLICK HERE TO EDIT) $<$}

Table I. These parameters have been implemented in Equation (7) to obtain $f_{\text {nom }}$ of $104.3 \mathrm{kHz}$. As there will be variations in $f_{\text {nom }}$ owing to the fabrication tolerances that might introduce imperfections, this value will be approximated to the experimental results. Besides, translation of spring coefficient in terms of Young's modulus of the material provides a clear explanation for the contribution of the springs shown in Fig. 2.

$$
k=\frac{4 \pi^{2} \beta^{2} T_{P}^{2} E m}{\rho L^{4}}
$$

The Young's modulus of aluminum, serving as the base material for the device linearly decreases with increase in temperature. Therefore, as seen in Fig. 8, the resonance frequency and the spring constant is seen to decrease with temperature owing to the dependence of the resonance frequency on Young's modulus of the device material, as shown in Equation (7). The device senses resonance frequency shifts due to temperature induced stress changes in the electrodes. Specifically, for small electrode-to-resonator gaps i.e. $2.5 \mu \mathrm{m}$, as in our case, a temperature induced $\mathrm{x}$-directed expansion causes a fractional change in $z_{0}$, leading to a change in the electrical spring stiffness $k_{e}$, which varies inversely proportional to $z_{0}^{3}$ and $V_{D C^{2}}{ }^{1}[24]$. This leads to a change in the resonant frequency governed by

$f_{0}=f_{\text {nоm }}\left\{1-\frac{V_{D C}^{2} \varepsilon_{0} T_{P} W}{2\left[z_{0}+\left(\alpha_{\text {sub }}-\alpha_{e}\right) L\left(T-T_{0}\right)\right]^{3} k_{m}}\right\}$

Here, $z_{0}$ is the electrode-to-resonator gap spacing at $T_{0}, k_{m}$ is the integrated mechanical stiffness of the resonator, $L$ and $W$ are the length and width of the movable plate respectively while $\alpha_{e}$ and $\alpha_{\text {sub }}$ are the thermal expansion coefficients of the electrode and substrate materials respectively [25]. Tungsten has been employed for the vias in the device membrane, and not in the suspension and anchors. Therefore, it has a negligible contribution in the device spring constant. Both the substrate and electrode is primarily made of Aluminum, for the reported device. The resonant frequency has been seen to decrease by $0.4 \mathrm{kHz}$, translated to a $k_{e}$ reduction of $1.73 \mathrm{~N} / \mathrm{m}$ with every increase in $20{ }^{\circ} \mathrm{C}$ from $-10{ }^{\circ} \mathrm{C}$ to $85{ }^{\circ} \mathrm{C}$. This owes to the decrease in Young's modulus of the material due to thermal expansion of the material with temperature rise. The TCE for aluminum has been obtained from the measurements with Equation (7) and Fig..8 as a reference. From the measurements, the $\mathrm{TCE}_{A L(E X P)}=-536.499 \mathrm{ppm} /{ }^{\circ} \mathrm{C}$ while the theoretical TCE of Aluminum $\left(\mathrm{TCE}_{A L \text { (Theoretical) }}\right)=-555.254 \mathrm{ppm} /{ }^{\circ} \mathrm{C}[22]$, with a convergence error of $3.15 \%$. As Aluminum forms the base material for our device structure, this helps in validating our device performance in terms of reliability.

Equation (9) can be differentiated with respect to temperature, followed by dividing by $f_{0}$, yields the temperature coefficient of the resonance frequency for this device. This can be expressed as

$$
T C f_{0}=\frac{1}{f_{0}} \frac{\partial f_{0}}{\partial T}
$$

For the case of our device, the temperature coefficient of resonant frequency $\left(T C f_{0}\right)$ has been calculated from the experimental measures shown in Fig. 8, which has a value of $268.612 \mathrm{ppm} /{ }^{\circ} \mathrm{C}$.

A quadratic polynomial is adopted to fit the experimental results for the device, by varying the differential frequency (ppm, which shows the first-order TCF of $-268.612 \mathrm{ppm} /{ }^{\circ} \mathrm{C}$ as well as a second-order TCF of $-0.306 \mathrm{ppb} /{ }^{\circ} \mathrm{C}^{2}$. The variations in resonant frequency and Young's modulus is shown as a function of variation in temperature to extract the TCF and TCE respectively in Fig. 9. This would provide some information for the compensation for the temperature drift due to the base material of the device i.e. Aluminum [25].

\section{Capacitance Measurements}

An important procedure for evaluating the thermal behavior of the device is the electrode-to-resonator gap variations with temperature. The force is proportional to the square of the voltage. Thus, a DC bias voltage is required to create the force, $F$ that excites the oscillator exactly at the AC driving voltage frequency [24]. The net capacitance is related to the DC bias on the resonator membrane in Equation (11):

$C V_{D C}^{2}=2 k\left(z_{0}-z\right) z$

where $C$ is the net active capacitance and $V_{D C}$ is the DC bias.

Fig. 10 shows the capacitance variations of the device when $V_{D C}$ is swept from $-20 \mathrm{~V}$ to $20 \mathrm{~V}$. As the $V_{D C}$ is increased, the force applied to the membrane increases resulting in decreased electrode-to-resonator gap, and increased capacitance. However, under application of very high DC bias, the effect of temperature becomes apparent, as the electrostatic springsoftening effect makes the results more visible. Besides, any movement of the plate is amplified close to the pull-in voltage. Any relative capacitance due to temperature becomes more noticeable with a higher temperature. As a result, a larger change in capacitance can be observed at a higher temperature. The capacitance has shown an increase from $270 \mathrm{fF}$ to $320 \mathrm{fF}$ at room temperature because of the reduced resonator-toelectrode gap owing to the increased bias from $0 \mathrm{~V}$ to $20 \mathrm{~V}$ over the movable resonator. This also helps us to set the DC bias to a nominal value of $10 \mathrm{~V}$ to avoid snapping of the plates i.e. occurrence of the pull-in effect.

Fig. 11, showing the variation of device capacitance with temperature. With bias voltages of $15 \mathrm{~V}$ to $20 \mathrm{~V}$, the static capacitance variations overcome the measurement noise and precision, revealing a higher capacitance at lower temperatures, hence a smaller electrode-to-resonator gap. For lower bias voltages of $5 \mathrm{~V}$ and $10 \mathrm{~V}$, the capacitance variation is below the measurement noise, indicated by the error bars. A lower bias is thereby preferred to a higher bias as there is higher chance of 


\section{$>$ REPLACE THIS LINE WITH YOUR PAPER IDENTIFICATION NUMBER (DOUBLE-CLICK HERE TO EDIT) $<$}

signal transfer in the latter case as the device moves to an unstable region and exhibits non-linearity under the effect of high bias voltages.

\section{Statistical Mismatch}

The release process was performed at different intervals to test the effect of etching time on the variation of resonance frequencies of the device, as shown in Fig. 12. Following the release of the device, the correlation between the etching time and resonance frequency was studied, where the resonance frequency was seen to drop with increased etching time. This effect is dominated by the under-etching of the aluminum layers, affecting the suspension of the optimized flexural membrane that advances with the etching time. It is worthwhile to note that use of $\mathrm{Al}$ as the base material for our device reduces the material losses while tungsten increases the device mass increasing the net $Q$. The effective $Q$ can be correlated to the mass of our device

$$
Q=\frac{m \omega_{0}}{c_{\text {total }}}
$$

where $Q$ is the effective quality factor, $\omega_{0}$ is the angular frequency, $m$ is the effective mass, and $c_{\text {total }}$ is the net damping coefficient of the resonator. As the mass of our device is dominated is by the use of tungsten for the vias, the device $Q$ primarily comes from the vias. The results, however, show that etching time is not very critical for the mechanical performance of the device. The relatively small coefficient of dispersion $\left(R^{2}\right.$ being 0.46) and dispersion of results of the variation of the resonance frequency of samples with etching time show that most probably other process-related phenomena affect the actual result, the residual stress variations being one of them. The details of this behavior are beyond the scope of this work.

A sum of twenty-five samples have been released at the same etching time i.e. $100 \mathrm{~min}$ and their characteristics have been studied at atmospheric pressure and room temperature. The histogram of Fig. 13 shows that the resonant-frequency range shown by the released resonators had a range of $100 \mathrm{kHz}$ to 105 $\mathrm{kHz}$. This depicts the maximum number of samples showing the same resonance hence the same frequency and spring constant. The standard deviation of the resonance frequency of samples etched at the same time has been observed to be around $7.0 \mathrm{kHz}$ while the mean resonance frequency was computed to be $102.5 \mathrm{kHz}$. On the other hand, the standard deviation of the spring constant was found to be $25.18 \mathrm{~N} / \mathrm{m}$ with a mean of 191.5 $\mathrm{N} / \mathrm{m}$.

\section{E. Characterization of nonlinearity}

The pressure sensor should be operated below the hysteresis point to obtain predictable performance. The study of nonlinearity is intended to estimate the maximum achievable DC Bias and AC oscillation voltage for this micro-sized sensor which is limited by the non-linear effects. It has been observed that as the AC oscillation voltage increases, the resonance frequency of the pressure sensor shifts from the linear resonance, turning it from a simple harmonic resonator with a linear restoring force into a Duffing resonator with hysteresis. These nonlinearities typically originate from either external nonlinear potentials or geometric effects [26]. When driven by an external sinusoidal force, the sensor behavior is described the equation of motion

$m \ddot{z}+b \dot{z}+k z+\beta z^{2}+\alpha z^{3}=0, \quad$ with $\beta>0, \alpha<0$

where $m$ is the effective mass, $k=m \omega_{0}^{2}$ is the effective spring constant, softened by the electrostatic attraction to the fixed electrode, $\beta$ is the positive symmetry-breaking quadratic elastic constant that pulls the sensor towards the fixed electrode regardless of the sign of $z, \alpha$ is the cubic spring constant or Duffing elastic constant that softens the effect of the linear restoring force owing to its negative sign [27], [28].

In the measurement of the phase-frequency curvse shown in Fig. 14, the pressure point is lowered to a point where the highest $\mathrm{AC}$ oscillation voltage i.e. $150 \mathrm{mV}$ drives the device into nonlinearity due to the high oscillation amplitude. The drive level is then lowered gradually and observed till the device shows a good linearity. Any point below this i.e. $30 \mathrm{mV}$ enables the device to work in its usable dynamic range.

\section{CONCLUSION}

The characterization results of a CMOS-MEMS flexural mode capacitive resonant pressure sensor over different conditions of pressure, temperature and parametric excitation have been presented. A $Q$-factor greater than 60 has been accomplished over the full-scale pressure range of $100 \mathrm{~Pa}-100$ $\mathrm{kPa}$ and a consumer grade temperature range of $-10{ }^{\circ} \mathrm{C}-85^{\circ} \mathrm{C}$. The device design has been optimized in terms of its $Q$ and sensitivity against device capacitance, in terms of Knudsen number such that the device performance is not independent of the surrounding pressure at atmospheric regime. The presented study involves the characterization of temperature and pressure along with effects of parametric excitations in evaluating the performance of the manufactured pressure sensor in terms of $Q$ and resonant frequency. The capacitive variations in the device have been studied in response to the electrical stimuli to identify the correct operating electrical conditions i.e. AC oscillation voltage and DC bias and conclude that a low level of DC bias is preferred owing to less signal transfer to the device The performed statistical study on twenty-five samples etched at the same time gives us knowledge about the device variations, allowing to estimate the device yield. This allows designing a commercially viable sensor with a low fabrication cost and maximum yield. In our case, Aluminum is the base material of our sensor, and in order to improve the TCE of the reported CMOS-MEMS sensor, the use of other materials in CMOS process with positive TCE could promise to offer a convenient passive temperature compensation scheme. This could help in compensating for the temperature drift i.e. setting the $\mathrm{TCf}_{0}=0$.

Since the unpackaged resonant sensor has been 


\section{$>$ REPLACE THIS LINE WITH YOUR PAPER IDENTIFICATION NUMBER (DOUBLE-CLICK HERE TO EDIT) $<$}

characterized, for each possible package, the characteristics can be modified depending on the introduced stress and other factors. Besides, MEMS parasitics, indicated by the antiresonant frequencies of the device, are second-order non-linear effects seem to make the integration of the device with the oscillator circuit very difficult. An on-going work of a complete cancellation of the MEMS parasitics of the sensor with a differential Low-Noise Amplifier is recognized as a future work.

The working prototype herein presented can be integrated as an altimeter or other potential applications into any wearable without any significant increase in cost or volume. On the whole, the reported characterization methodology will allow a designer working with resonators, to account for several aspects, from device design optimization to determining the optimal conditions for device operation.

\section{REFERENCES}

[1] H. Van Heeren and P. Salomon, "MEMS - Recent Developments, Future Directions," Electron. Enabled Prod. Technol. Watch, pp. 1$51,2007$.

[2] H. Qu, "CMOS MEMS fabrication technologies and devices," Micromachines, vol. 7, no. 1, pp. 1-21, 2016.

[3] G. K. Fedder, "CMOS-based sensors," Proc. IEEE Sensors, vol. 2005, pp. 125-128, 2005.

[4] C.-L. C. and H.-C. C. and C.-I. C. and W. Fang, "Development of a CMOS MEMS pressure sensor with a mechanical force-displacement transduction structure," J. Micromechanics Microengineering, vol. 25, no. 12, p. 125024, 2015.

[5] Y. Hui, T. Nan, N. X. Sun, and M. Rinaldi, "High resolution magnetometer based on a high frequency magnetoelectric MEMSCMOS oscillator," J. Microelectromechanical Syst., vol. 24, no. 1, pp. 134-143, 2015.

[6] M. Narducci, L. Yu-Chia, W. Fang, and J. Tsai, "CMOS MEMS capacitive absolute pressure sensor," J. Micromechanics Microengineering, vol. 23, no. 5, p. 55007, 2013.

[7] Y. Ahn, S. Song, and K.-S. Yun, "Woven flexible textile structure for wearable power-generating tactile sensor array," Smart Mater. Struct., vol. 24, no. 7, p. 75002, 2015.

[8] S. Ren, W. Yuan, D. Qiao, J. Deng, and X. Sun, "Pressure sensor with integrated resonator operating at atmospheric pressure," Sensors (Switzerland), vol. 13, no. 12, pp. 17006-17024, 2013.

[9] J. A. Montiel-Nelson, J. Sosa, R. Pulido, A. Beriain, H. Solar, and R. Berenguer, "Digital output MEMS pressure sensor using capacitanceto-time converter," Proc. 2014 29th Conf. Des. Circuits Integr. Syst. DCIS 2014, pp. 1-4, 2014.

[10] A. D. Sundararajan and S. M. R. Hasan, "Elliptic diaphragm capacitive pressure sensor and signal conditioning circuit fabricated in SiGe CMOS integrated MEMS," IEEE Sens. J., vol. 15, no. 3, pp. 1825-1837, 2015.

[11] F. Y. Kuo, C. Y. Lin, P. C. Chuang, C. L. Chie, Y. L. Yeh, and K.-A Wen, "Monolithic Multi-sensor Design with Resonator-Based MEMS Structures," IEEE J. Electron Devices Soc., vol. 6734, no. c, pp. 1-1, 2017.

[12] S. Banerji, J. Madrenas, and D. Fernandez, "Optimization of parameters for CMOS MEMS resonant pressure sensors," Symp. Des. Test, Integr. Packag. MEMS/MOEMS, DTIP 2015, pp. 1-6, 2015.

[13] S. Banerji, P. Michalik, D. Fernández, J. Madrenas, A. Mola, and J. Montanyà, "CMOS-MEMS resonant pressure sensors: optimization and validation through comparative analysis," Microsyst. Technol., pp. 1-17, 2016.

[14] A. K. Pandey, R. Pratap, and F. S. Chau, "Effect of pressure on fluid damping in MEMS torsional resonators with flow ranging from continuum to molecular regime," Exp. Mech., vol. 48, no. 1, pp. 91106,2008

[15] D. Fernández, P. Michalik, J. Madrenas, and J. M. Sánchez-Chiva,
“CMOS BEOL-embedded z-axis accelerometer," Electron. Lett., vol. 51, no. 11, pp. 865-867, 2015.

[16] D. Fernández, J. Ricart, and J. Madrenas, "Experiments on the release of CMOS-micromachined metal layers," J. Sensors, vol. 2010, 2010.

[17] S. Banerji, J. Madrenas, and D. Fernandez, "Temperature and pressure characterization of the quality factor in a CMOS-MEMS resonator," 2016 Ieee Sensors, no. c, pp. 1-3, 2016.

[18] B. Kim et al., "Temperature dependence of quality factor in MEMS resonators," J. Microelectromechanical Syst., vol. 17, no. 3, pp. 755766, 2008.

[19] J. O. Dennis, A. Y. Ahmed, and M. H. Khir, "Fabrication and characterization of a CMOS-MEMS humidity sensor," Sensors (Switzerland), vol. 15, no. 7, pp. 16674-16687, 2015.

[20] M. A. Hopcroft et al., "Using the temperature dependence of resonator quality factor as a thermometer," Appl. Phys. Lett., vol. 91, no. 1, pp. 3-5, 2007.

[21] M. T. Jan, F. Ahmad, N. H. B. Hamid, M. H. B. Md Khir, K. Ashraf, and M. Shoaib, "Temperature dependent Young's modulus and quality factor of CMOS-MEMS resonator: Modelling and experimental approach," Microelectron. Reliab., vol. 57, no. December, pp. 64-70, 2016.

[22] "Young's Modulus of Elasticity for Metals and Alloys.".

[23] V. Kaajakari, "MEMS Tutorial: Pull-In Voltage in Electrostatic Microactuators," Pract. MEMS, no. 5, pp. 1-2, 2009.

[24] W.-T. Hsu, J. R. Clark, and C. T.-C. Nguyen, "A Resonant Temperature Sensor Based on Electrical Spring Softening," in IEEE International Solid-State Sensors and Actuators Conference (Transducers), 2001, vol. 2, pp. 1484-1487.

[25] V. Thakar and M. Rais-Zadeh, "Temperature-compensated piezoelectrically actuated Lam??-mode resonators," in Proceedings of the IEEE International Conference on Micro Electro Mechanical Systems (MEMS), 2014, pp. 214-217.

[26] S. Tadigadapa and N. Najafi, " Reliability of micro-electromechanical systems (MEMS)," Proc. SPIE, vol. 4558, pp. 197205,2001 .

[27] R. Lifshitz and M. C. Cross, "Nonlinear Dynamics of Nanomechanical and Micromechanical Resonators," Rev. Nonlinear Dyn. Complex., pp. 1-52, 2009.

[28] V. Kaajakari, T. Mattila, A. Oja, and H. Seppä, "Nonlinear limits for single-crystal silicon microresonators," J. Microelectromechanical Syst., vol. 13, no. 5, pp. 715-724, 2004.

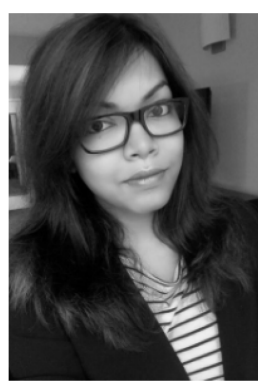

Saoni Banerji was born in Bokaro Steel City, India, in 1989. She received the B.Tech. degree in Electrical and Electronics engineering in 2011, M.Sc. degree in Electronics from Nanyang Technological University, Singapore in 2014, and Diploma in Business Management from London School of Business and Finance (LSBF) in April 2017. She is currently pursuing her $\mathrm{PhD}$ in the fields of MEMS design and circuits, and designing control architectures for MEMS Sensors signal conditioning in Universitat Politècnica de Catalunya (UPC), Barcelona, Spain. Her current research interests and competencies include MEMS modeling, design of signal conditioning architectures with CMOS processes, testing and characterization of CMOSMEMS devices. Since August 2017, she is working in Nanusens, gaining experience with finite element simulations, characterization and measurements of CMOS-MEMS accelerometers. 


\section{$>$ REPLACE THIS LINE WITH YOUR PAPER IDENTIFICATION NUMBER (DOUBLE-CLICK HERE TO EDIT) $<$}

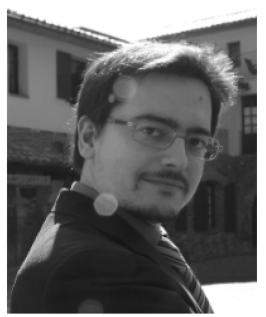

Daniel Fernández was born in Barcelona, Spain, in 1979. He received the M.Sc. degree in telecommunications engineering, Ph.D. degree (cum laude), and M.B.A. degree from Universitat Politècnica de Catalunya (UPC), Barcelona, Spain, in 2004, 2008 and 2009, respectively. From 2008 to 2010 he worked as a Postdoctoral Researcher at the Electronic Engineering Department, UPC in the fields of CMOS surface micromachining, circuits and control architectures for MEMS sensors and actuators, trans linear circuits for analog signal processing and digital implementations of power converters. From 2010 to 2014 he worked as Principal ASIC Engineer at Baolab Microsystems developing circuits and architectures for CMOS MEMS/NEMS-based products, and as ASIC Design Engineer Contractor for the European Space Agency designing radiation-hardened integrated-circuits and interface blocks for space exploration in interplanetary missions. Currently he is Chief Technology and Science Officer at Nanusens, Bellaterra, Spain, where he works towards the development of innovative circuits and architectures for MEMS sensors signal

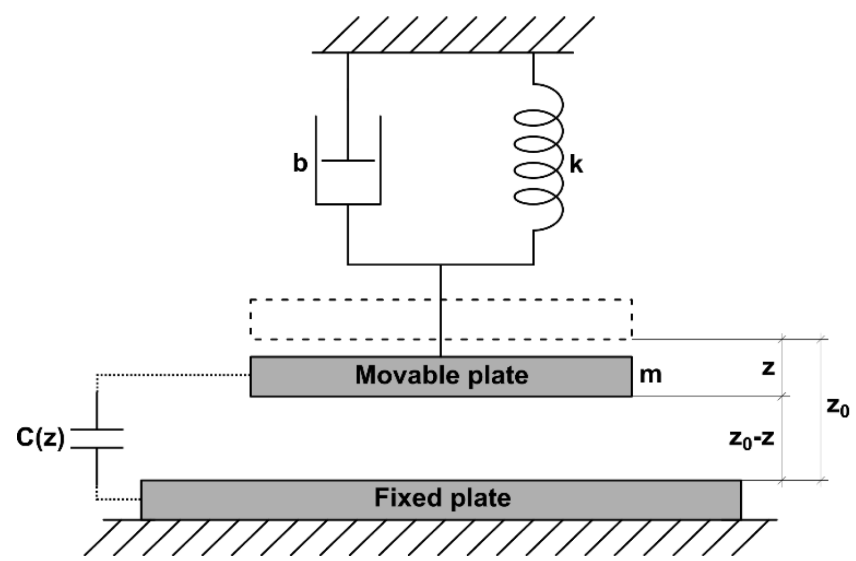

Fig. 1. Lumped model of the two plate CMOS-MEMS pressure sensor

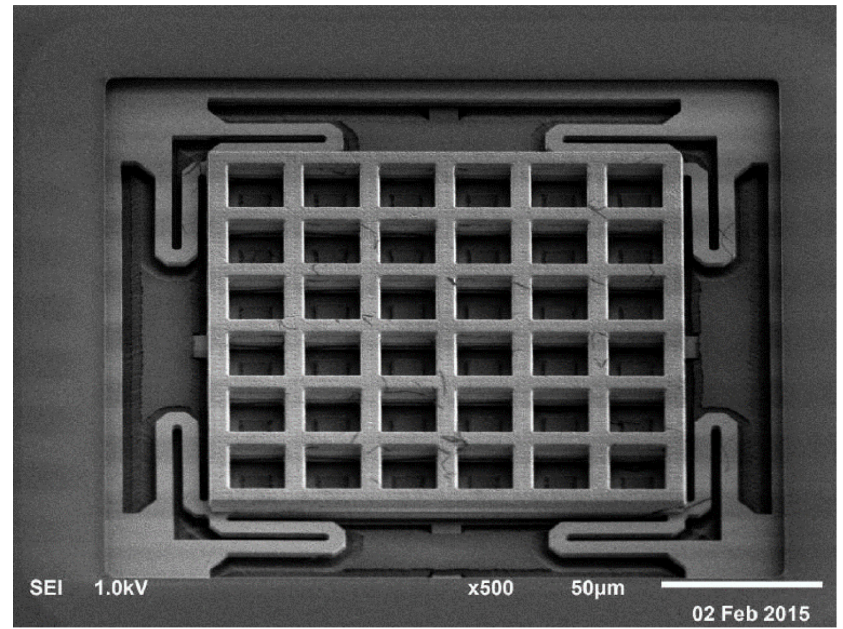

Fig. 2. Scanning Electron Microscope (SEM) micrograph of the fabricated CMOS pressure sensor conditioning.

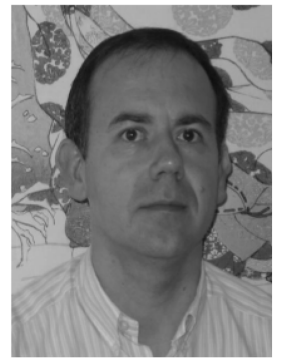

Jordi Madrenas (Telecom. Eng. '86, Ph.D. '91, UPC, Barcelona, Catalunya, Spain) is currently Associate Professor at the Department of Electronic Engineering, Technical University of Catalunya (UPC BarcelonaTech). He has participated in 5 European projects and coordinated 6 Spanish national research projects. Also, he has coordinated several contracts with companies. At present, he coordinates a national project on MEMS on-chip and microsensor bio-inspired signal processing. He has co-authored more than 150 scientific journal and international conference papers, two books and 5 book chapters. Between 2000 and 2003 he was Vice-Dean of Studies of the Telecommunication Engineering School of Barcelona, UPC. His current research interests include analog, mixedsignal and digital VLSI and FPGA design, CMOS-MEMS design and conditioning, ultra-low-power design, bioinspired/neuromorphic system implementation and rad-hard mixed-signal circuits. 


\section{$>$ REPLACE THIS LINE WITH YOUR PAPER IDENTIFICATION NUMBER (DOUBLE-CLICK HERE TO EDIT) $<$}

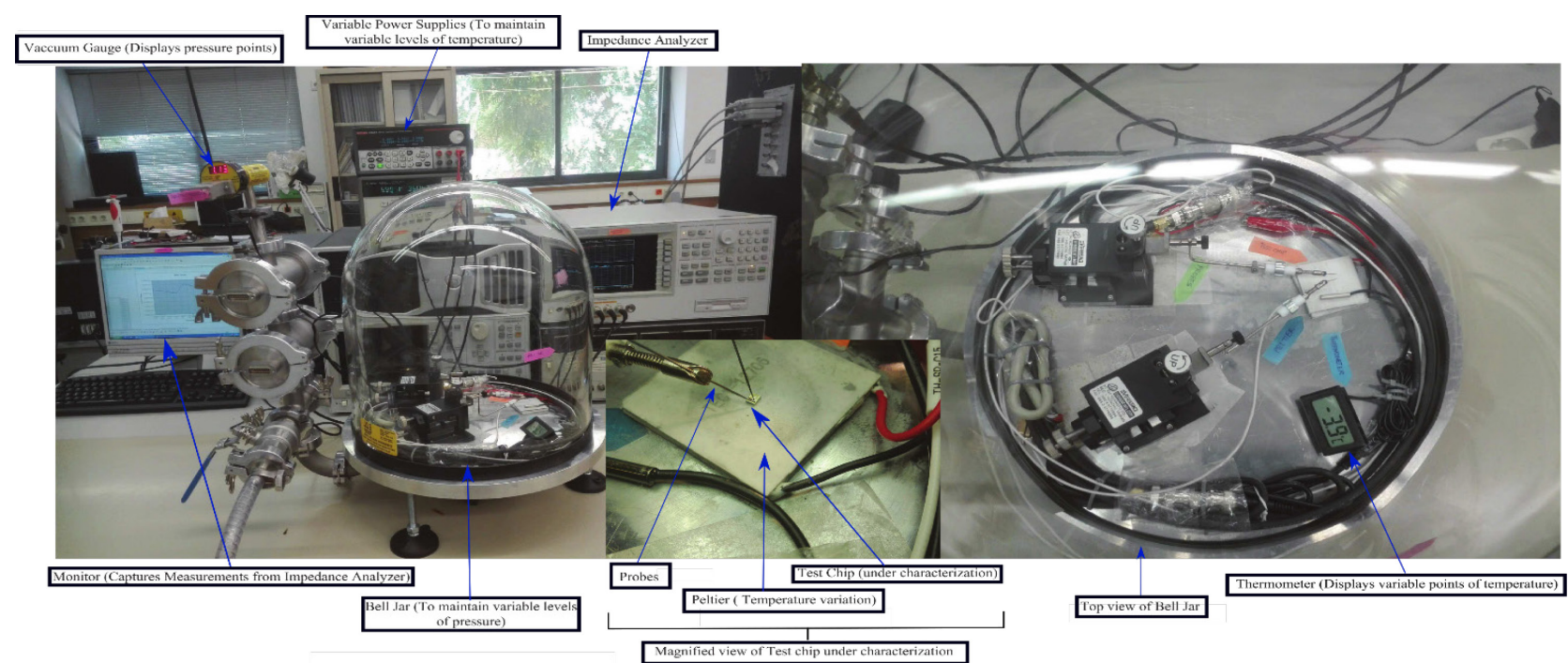

Fig. 3. Experimental set-up for characterization of the CMOS-MEMS pressure sensor

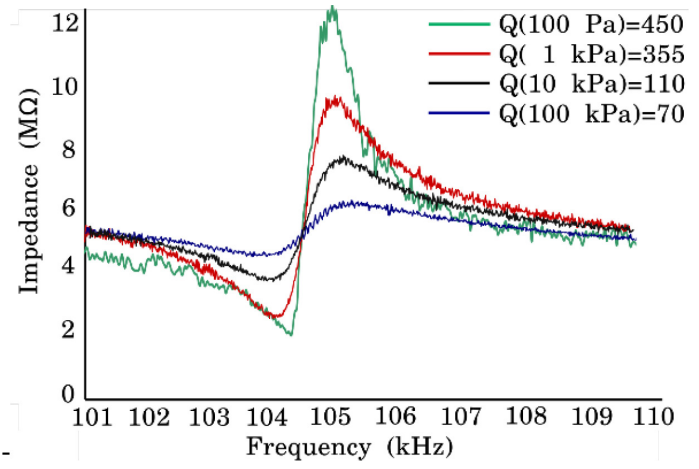

Fig. 4. Frequency response characteristics of the CMOS-MEMS pressure sensor illustrating the variation of Quality factor $(Q)$ under variable pressure regimes at room temperature and $100 \mathrm{~Pa}, 1000 \mathrm{~Pa}, 10 \mathrm{kPa}$ and $100 \mathrm{kPa}$.

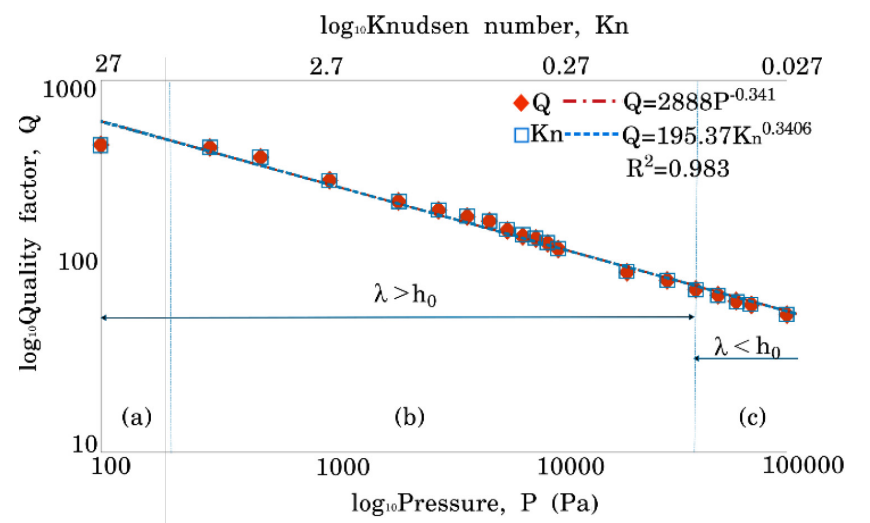

Fig. 5. Fitting of the experimental frequency response of the CMOS-MEMS pressure sensor to illustrate the effect of pressure on $Q$ at room temperature under variable pressures. $K_{n}$ scale is shown at the top over (a) molecular flow regime (b) transition flow regime (c) slip flow regime. $\left(K_{n}\right.$ is used for theoretical characterization of pressure regimes depending on the device gap). 


\section{$>$ REPLACE THIS LINE WITH YOUR PAPER IDENTIFICATION NUMBER (DOUBLE-CLICK HERE TO EDIT) $<$}

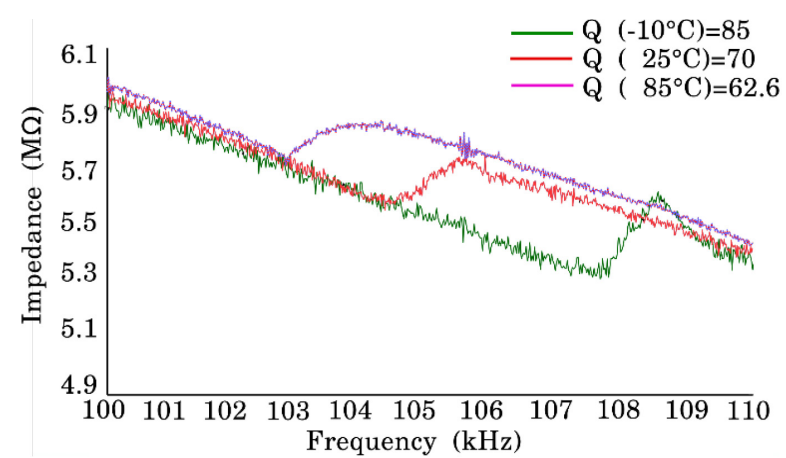

Fig. 6. Pressure sensor impedance module as a function of frequency illustrating the variation of the Quality factor $(Q)$ at $10 \mathrm{kPa}$ for $-10{ }^{\circ} \mathrm{C}, 25^{\circ} \mathrm{C}$ and $85^{\circ} \mathrm{C}$ temperatures.

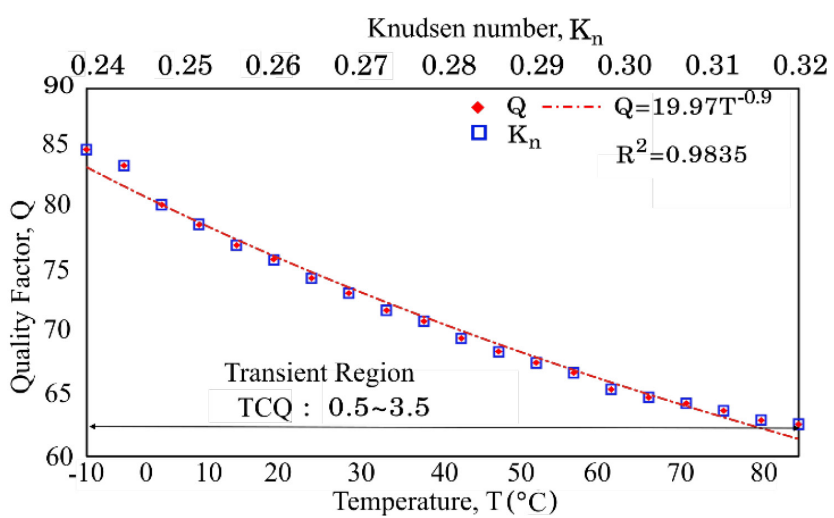

Fig. 7. Fitting of the experimental frequency response of the CMOS MEMS pressure sensor to illustrate the effect of temperature on $Q$ at $10 \mathrm{kPa}$ under variable temperatures

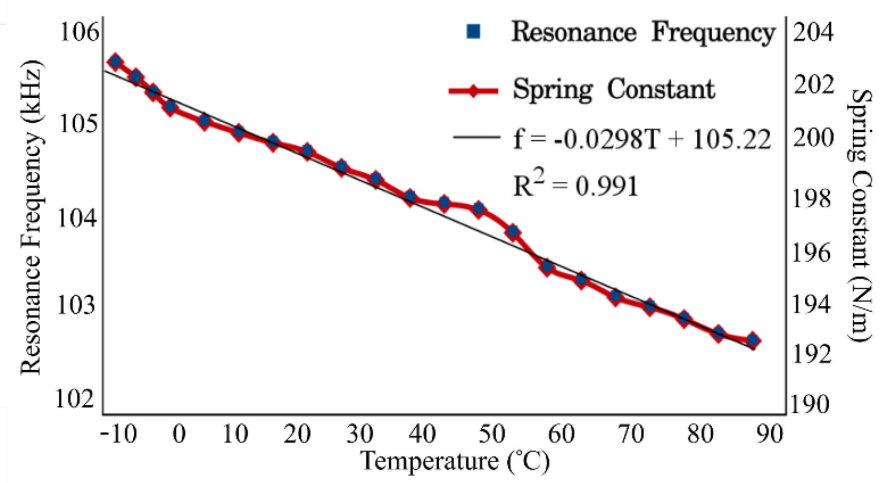

Fig. 8. Spring softening effect of temperature on resonance frequency 


\section{$>$ REPLACE THIS LINE WITH YOUR PAPER IDENTIFICATION NUMBER (DOUBLE-CLICK HERE TO EDIT) $<$}

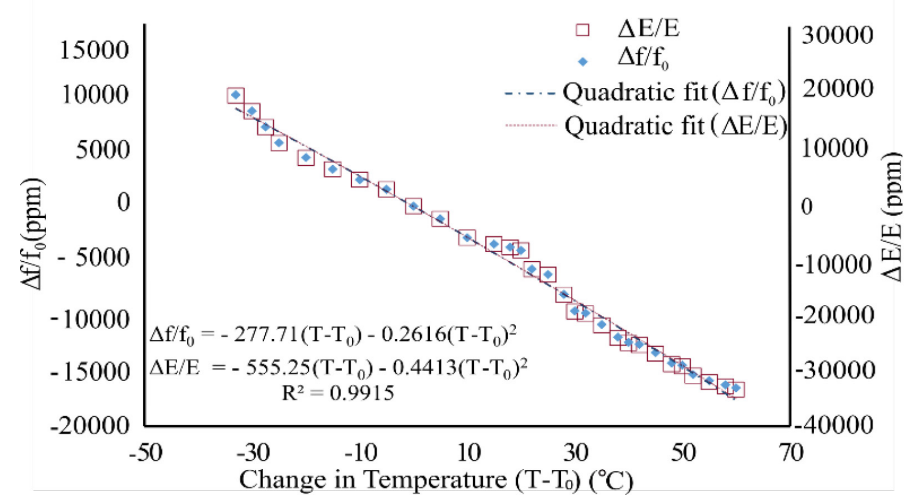

Fig. 9. Variation in resonance frequency and Young's modulus (ppm) as a function of change in temperature

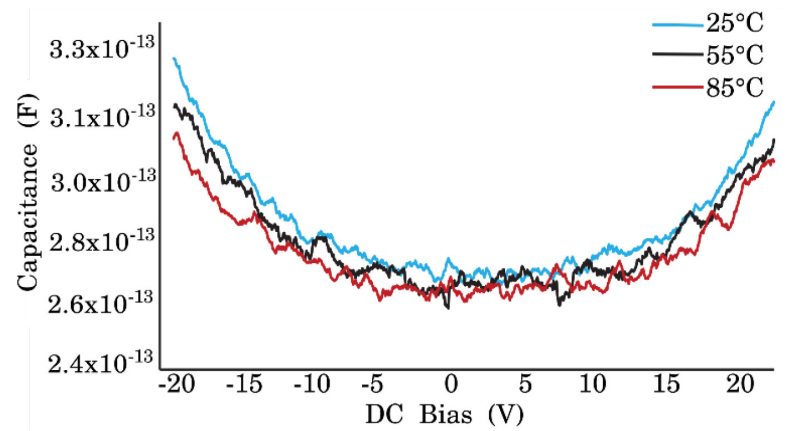

Fig. 10. Variation of capacitance of the pressure sensor with DC Bias

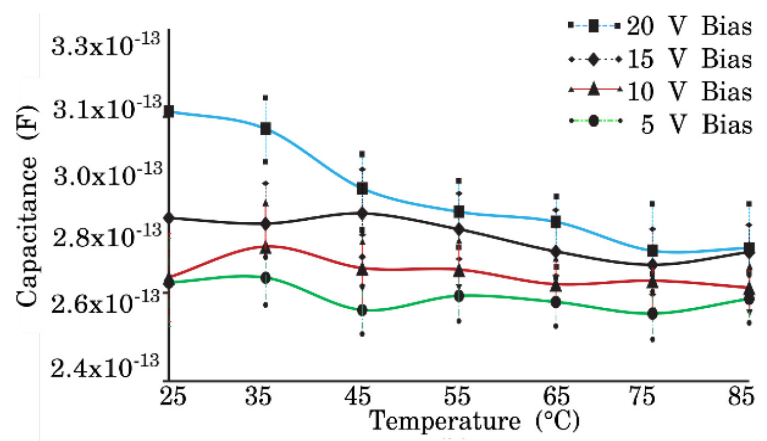

Fig. 11. Variation of capacitance of the pressure sensor with temperature

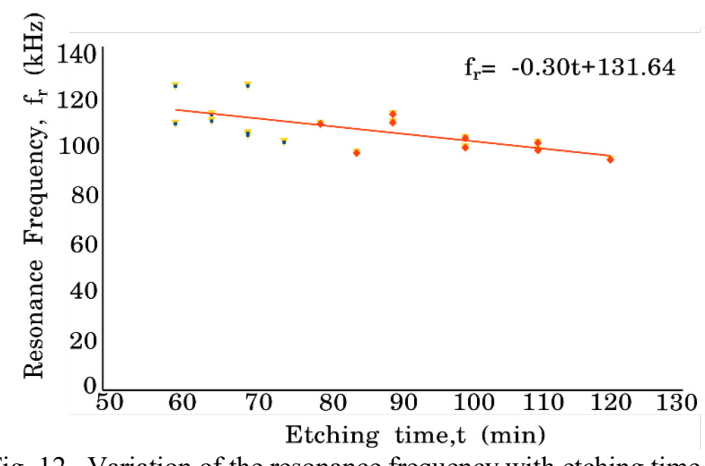

Fig. 12. Variation of the resonance frequency with etching time for different device samples 


\section{$>$ REPLACE THIS LINE WITH YOUR PAPER IDENTIFICATION NUMBER (DOUBLE-CLICK HERE TO EDIT) $<$}

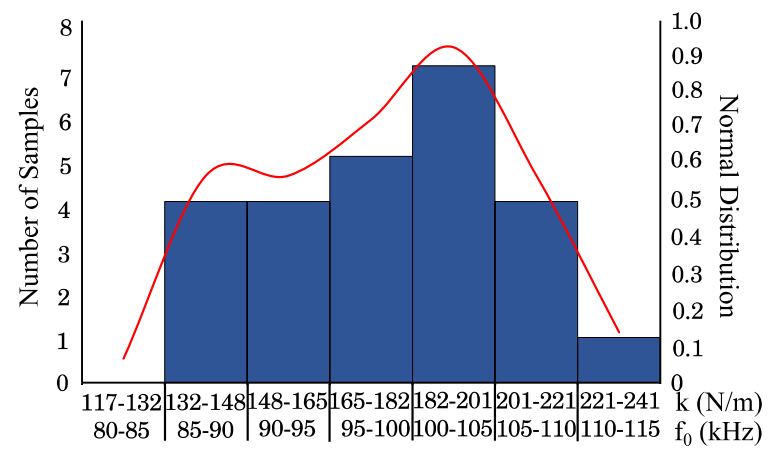

Fig. 13. Histogram the variability of the pressure sensor resonant frequency. All samples were released with the same etching time.

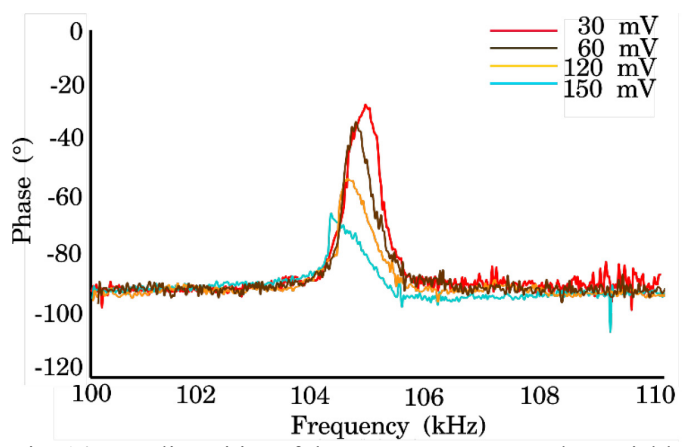

Fig. 14. Nonlinearities of the pressure sensor under variable drive levels 